\title{
Condições Afetivo-Emocionais em Mulheres com Síndrome Pré-Menstrual Através do Z-Teste e do IDATE ${ }^{1}$
}

\author{
Ronald Maeso Montes ${ }^{2}$ e Cícero Emidio Vaz \\ Pontifícia Universidade Católica do Rio Grande do Sul
}

\begin{abstract}
RESUMO - A presente pesquisa visou constatar as condições afetivo-emocionais em mulheres com síndrome pré-menstrual (SPM), comparadas com outras mulheres sem sintomas pré-menstruais. A amostra foi constituída de 43 universitárias, na faixa etária de 18 e 35 anos, distribuídas em dois grupos: Grupo 1, constituído de 25 mulheres com SPM, e Grupo 2 (Controle), de 18 mulheres sem estas disfunções. Foram utilizados como instrumentos a técnica de Zulliger (Z-Teste forma coletiva) e o Inventário de Ansiedade-Traço-Estado - IDATE (Spielberger), e para tratamento estatístico na análise comparativa dos dados o t-Teste para amostras independentes. O nível de significância escolhido foi $p \leq 0.05$. Os resultados indicaram que as mulheres do grupo com síndrome pré-menstrual reagem emocionalmente de forma mais intensa e têm tendência à perda de controle emocional em índice maior que as mulheres do grupo sem sintomas pré-menstruais.
\end{abstract}

Palavras-chave: síndrome pré-menstrual; condições afetivo-emocionais; controle emocional; Z-Teste; IDATE.

\section{Affective and Emotional Conditions in Women with Premestrual Syndrome on the Z-Test and IDATE}

\begin{abstract}
The present research aimed to investigate the affective-emotional conditions in women suffering of premenstrual syndrome, compared with others without premenstrual syndrome. Forty three university students, between 18 and 45 years old, formed the sample. The subjects were divided in two different groups by the following way: Group 1, with 25 women suffering premenstrual syndrome, and Group 2, with 18 women without this symptoms. It was used as set of instruments the Zulliger technique (T-Test) and Spielberger State Trait Anxiety Inventory (STAI); it was used the t-Test for independent.samples, for comparative analysis of data between two different groups. The significant level of acceptance chosen was $p \leq .05$. The results indicate that the group of women with premenstrual syndrome react with more intensity of emotion, losing the emotional control, than the group of women without these symptoms.
\end{abstract}

Key words: premenstrual syndrome; affective conditions; emotional conditions; emotional control; Z-Teste; IDATE.

A síndrome pré-menstrual (SPM), também conhecida como tensão pré-menstrual, de acordo com Verri e cols. (1997), corresponde a um conjunto de sintomas clínicos moderados como sensação de dolorimento nos seios, corpo edemaciado, cefaléia, mudanças no apetite, no comportamento alimentar e concomitante mudança no humor, enquanto ocorrem as modificações decorrentes da fase hormonal lútea tardia. Para Nogueira e Silva (2000), o quadro clínico da SPM é de sintomas diversificados, constituído, na maior parte das vezes, por irritabilidade, depressão, cansaço, cefaléia ou dores nas mamas, coexistindo sintomas físicos e psíquicos, durante três a sete dias, e de causa aparentemente multifatorial. A formação do corpo lúteo é necessária para a presença dos sintomas, porém o papel dos hormônios lúteos ainda não é claro. Estes sintomas acontecem repetidamente de forma cíclica antes da menstruação, diminuindo ou desaparecendo no final do ciclo. De acordo com Mendonça Lima e Camus (1996), os principais fatores de risco para esta síndrome são a idade superior a 30 anos, fluxo menstrual abundante, a hereditariedade e a história de vários ciclos menstruais não interrompidos por gravidez. A irritabilidade é o principal sintoma observado, ocorrendo em freqüência que varia de 3 a $9 \%$ dos sintomas pré-menstruais.

1 Trabalho realizado com o suporte do CNPq e da CAPES.

2 Endereço: Rua Marcelo Gama, 178. 90540-040, Porto Alegre, RS, Brasil. E-mail: raimm@terra.com.br
A SPM tem sido caracterizada, conforme vários autores (Halbreich, 1997 \& Cahill, 1998; Richardson, 1995; Rosales, Guerrero \& Moreno, 1996; Severino \& Moline, 1995; Walker, 1995), por ainda carecer de definição, etiologia e tratamento consensuais entre pesquisadores da área da saúde. Segundo Gurevich (1995), a SPM é uma entidade biomédica que envolve complexidade de fenômenos, sendo também uma categoria de construto ideológico e cultural que envolve uma série de crenças sobre a sua natureza. Apresenta-se com manifestações variadas, necessitando de critérios para a sua caracterização. Para Barnhart, Freeman e Sondheimer (1995), a SPM é um grupo de sintomas pré-menstruais predominantemente afetivos que desorganizam o comportamento rotineiro das mulheres, necessitando que o profissional diferencie este diagnóstico de desordens afetivas maiores, ou de outros sintomas que não provoquem desorganização na menstruação.

Bancroft (1995) aponta três fatores importantes na SPM. O primeiro deles é a própria menstruação interferindo no humor da mulher e no seu bem estar na fase pré-menstrual, assim como durante a menstruação. O segundo fator envolve as alterações do ciclo hormonal, produzindo variabilidade cíclica em certos aspectos no funcionamento do sistema nervoso central. O terceiro fator é a vulnerabilidade de algumas mulheres ao responder de forma desproporcionada, física e emocionalmente a agentes adversos. No entender desse autor, existem evidências de que as mudanças de humor, freqüente- 
mente encontradas na SPM, estão ligadas a alterações cíclicas da atividade serotoninérgica do sistema nervoso central, e os desejos alimentares pré-menstruais seriam manifestações destas alterações.

Segundo os critérios de Halbreich (1997a), qualquer sintoma físico, transtorno de humor ou comportamental poderá ser considerado como SPM, quando for recorrente e cíclico, relacionado ao período folicular do ciclo, causando angústia ou prejuízo nas atividades rotineiras diárias. $\mathrm{O}$ autor acrescenta que esses sintomas devem ser documentados em diário para monitoramento sintomatológico.

\section{Prevalência da SPM}

A prevalência da SPM tem se apresentado com grande variação de achados. Reid e Yen (1983) estimaram que $80 \%$ das mulheres sentem desconforto físico e apresentam alteração de humor no período pré-menstrual. Lima e Camus (1996) acreditam que $95 \%$ das mulheres férteis sentem pelo menos um sintoma leve, e $11 \%$ se sentem incapacitadas de executar suas rotinas diárias. Daugherty (1998), Melo, Giribela, Giribela e Ricci (2001) estimam que $40 \%$ das mulheres em idade reprodutiva tenham SPM e que 5\% destas a apresente de forma mais severa, referindo não haver um teste diagnóstico específico para esta síndrome. Mais recentemente, Wyatt, Dimmock, Frischer, Jones e O'Brien (2002) referem que entre $85 \%$ a $97 \%$ de mulheres em idade reprodutiva sofrem de sintomas variados no período pré-menstrual, e que $30 \%$ a $40 \%$ destas procuram atendimento médico.

\section{Aspectos psicológicos da Síndrome Pré-Menstrual}

As publicações que tratam do tema síndrome pré-menstrual apresentam extensa variação de abordagens sobre condições emocionais e outros aspectos psicológicos relevantes, que nos têm permitido conhecer suas particularidades.

Os sintomas pré-menstruais, segundo Halbreich (1997a), são uma expressão de traços vulneráveis de personalidade que surgem em resposta a determinado estímulo desencadeante. Para o autor, a forma como estes sintomas pré-menstruais se apresentam é expressa através destes traços de personalidade, que tem, por vezes, natureza depressiva e de ansiedade. Mortola (1992) apresenta dois modelos teóricos para justificar a SPM: a) deficiência de progesterona; b) modelo psicogênico induzido pelo estresse. $\mathrm{O}$ primeiro modelo tem por base a deficiência de progesterona como fator desencadeante da SPM. Este modelo foi utilizado por aproximadamente uma década, através da utilização de progesterona intravaginal em mulheres com SPM. No entanto, a falta de critérios adequados de controle não permitiu que se conseguisse determinar sua eficácia, apesar da progesterona ser, até o momento, bastante prescrita para no tratamento da SPM. No modelo psicogênico, Mortola descreve a SPM como fruto de vivências de eventos estressantes em mulheres com características de personalidade histriônica. De acordo com Wyatt, Dimmock, Jones, Obhrai e O'Brien (2001), não existem evidências científicas que justifiquem a necessidade da prescrição de progesterona no manejo clínico dos sintomas de SPM.

Para Facchinetti, Tarabusi e Nappi (1998), a SPM é uma desordem psicobiológica freqüentemente diagnosticada como ansiedade. Salientam os autores que a SPM necessita apresentar pelo menos um sintoma emocional suficientemente severo para prejudicar a rotina social, não sendo mera exacerbação de um sintoma psiquiátrico. Acreditam que o período pré-menstrual pode predispor determinadas mulheres a uma má adaptação a agentes estressores.

De acordo com as observações de Halbe (1987), uma das possíveis etiologias de alguns sintomas da SPM seria a expectativa da menstruação mobilizando conflitos e frustrações inconscientes, relacionados ao desejo ou a rejeição à procriação. Esta tese, embora sendo um construto compreensível, não foi completamente aceita no meio médico, porque, além da já conhecida participação hormonal na sintomatologia, sua fundamentação é originada a partir de observações pessoais de pacientes, sendo fonte de vieses de seleção e aferição.

Estudos realizados por Glick, Endicott e Nee (1993) e Condon (1993), com famílias e com gêmeos, sugerem que fatores familiares influenciam na vulnerabilidade individual dos sintomas da SPM. Porém, conforme Kendler, Karkowski, Corey e Neale (1998), estas pesquisas não conseguiram justificar a fidedignidade de seus resultados, além do fato de se conhecer muito pouco sobre a relação dos fatores de risco familiares para a SPM e depressão maior. Para Van den Akker, Eves, Stein e Murray (1995), existem contribuições genéricas para os sintomas relacionados SPM; entendem esses autores que sintomas do tipo extroversão, neuroticismo e depresão são condições emocionais comuns nesta sídrome.

Segundo O’Brien (1997), entre os problemas clínicos principais da SPM estão a identificação e o diagnótico corretos, devendo-se diferenciar nas pacientes que sofrem da verdadeira SPM, sintomas psicológicos considerados comuns provocando sintomas orgânicos, psicopatologias coexistindo com SPM, ou psicopatologias desencadeadas pela SPM.

Fazendo referência à crença social de que mulheres com SPM não controlam seu humor, seu processo de cognição e comportamento, devido à flutuação dos níveis hormonais, Choi e McKeown (1997) analisaram a influência social deste estereótipo; entrevistaram nove estudantes do sexo feminino sobre suas experiências com ciclos menstruais, utilizando a análise qualitativa para a distinção entre suas individualidades e suas experiências pré-menstruais. Os autores verificaram que as experiências referidas com relação a sintomas menstruais pelas mulheres da amostra foram menos incidentes do que seus conceitos prévios sobre SPM. Concluíram que as mulheres podem distinguir entre experiências menstruais próprias e estereótipos pré-menstruais construídos na sociedade. Em pesquisa semelhante, com maior amostragem, realizada na Islândia, Sveinsdottir (1998) avaliou o diário prospectivo de saúde de 83 mulheres, durante 211 ciclos menstruais, e verificou que apenas $8,4 \%$ delas preenchiam os critérios diagnósticos para SPM, índice bem inferior ao encontrado em estudos americanos. Concluiu que os sintomas desta síndrome possivelmente sofrem influências sociais, no que tange a referências e a expectativas.

Os fatores relacionados à depressão, conforme Freeman, Rickels, Schweizer e Ting (1995), estão associados à intensidade dos sintomas da SPM, sendo a faixa etária dos 20 aos 35 anos de idade mais vulnerável à ansiedade neste transtorno. Na China, Chau e Chang (1998) examinaram a relação entre ansiedade e SPM em 153 jovens em idade escolar utilizando 
o Inventário de Ansiedade Traço-Estado (IDATE) de Spielberger, Gorsuch e Lushene (1979) e o Abraham's Menstrual Symptom Questionnaire. Foi encontrando alto nível de ansiedade traço nesta amostra, correlacionado com o aumento da intensidade dos sintomas da SPM. Yu, Zhu, Li, Oakley e Reame (1996) exploraram os sintomas pré-menstruais de 16 chinesas de área urbana do sudeste asiático, usando um questionário e diários específicos, modificados pelo fator cultural. Durante a fase pré-menstrual, verificaram aumento significativo em sintomas como fadiga, sensação de frio, dor e inchaço nos seios, sonolência, dor ou desconforto abdominal e diminuição do desejo sexual. Notaram, todavia, discrepância em alguns resultados relacionados a sintomas emocionais. Um deles é o de que através do Menstrual Distress Questionnaire, 13 e $25 \%$ das mulheres vivenciaram flutuações severas no humor e irritabilidade durante as fases pré-menstrual e menstrual. Entretanto, não houve correlação significativa entre estes sintomas e o ciclo menstrual. Concluíram que a angústia ocorrida no período perimenstrual em mulheres chinesas pode sofrer a interferência do procedimento na coleta de dados. Esta mesma pesquisa faz referência à investigação transcultural realizada pela World Health Organization em 1981, com 5300 mulheres, de 14 grupos culturais de dez países, revelando que, embora as mulheres apresentassem sintomas físicos e psicológicos negativos além de mudanças no comportamento relacionadas à menstruação, apresentaram variações de sintomas, desde $23 \%$ e $34 \%$ em dois grupos da Indonésia, até $71 \%$ e $73 \%$ no Reino Unido e na Iugoslávia, respectivamente. Os grupos norte-americano e italiano apresentaram prevalências muito semelhantes.

Van den Akker, Eves, Service e Lennon (1995) pesquisaram a influência cultural sobre o comportamento, através das diferentes fases do ciclo menstrual, em três grupos étnicos habitantes da Inglaterra. Participaram da amostra 43 afro-caribenhas, 73 caucasianas e 32 orientais submetidas ao Positive, Negative Affectivity Scale, uma estimativa retrospectiva dos sintomas pré-menstruais com informações dos sintomas diários durante 35 dias. O grupo caucasiano apresentou níveis significativamente mais elevados de sintomas pré-menstruais e menstruais, quando comparado com os outros dois grupos. A análise das escalas internas deste instrumento sugeriu elevação nos sintomas referidos em decorrência do humor, sintomas físicos e dor, e não por performance mental e comportamento social. A descrição dos sintomas e a baixa afetividade na fase intermenstrual não apresentaram diferença significativa entre os três grupos.

A relação entre SPM e depressão é sugerida por Pearlstein (1995), pela crescente prevalência de episódios depressivos e pela eficácia do tratamento com antidepressivos em mulheres com esta síndrome. Contudo, convém lembrar que, para Yonkers e Chantilis (1995), Bhatia e Bhatia (1999), as mulheres, independentemente de SPM, apresentam mais depressão do que os homens, na proporção de 2:1, sendo que a prevalência de depressão maior em mulheres é estimada em 21\%. Para Halbreich (2001), os períodos de flutuação hormonal ou de instabilidade dos estrógenos têm relação com aumentos na vulnerabilidade à depressão entre mulheres suscetíveis. Para o autor, é possível que o fenótipo deste tipo de depressão seja distinto das depressões não ligadas a eventos reprodutivos, ou das que acometem os homens.
Com o objetivo de analisar a estabilidade dos sintomas em mulheres com esta síndrome, Bloch, Schmidt e Rubinow (1997) investigaram 16 mulheres sintomáticas e as acompanharam por mais de dois ciclos menstruais. Verificaram que os sintomas mais estáveis neste grupo foram ansiedade, irritabilidade e labilidade emocional, concluindo que a SPM pode ser mais bem compreendida como uma face das desordens recorrentes de humor.

Tendo como objetivo investigar depressão e ansiedade através do Inventário de Depressão de Beck, o Automatic Thougts Questionnaire e o Inventário de Ansiedade EstadoTraço (IDATE), Christensen e Oei (1995) realizaram estudo comparativo que contou com a participação de pacientes, sendo 32 mulheres não tratadas para SPM e 52 tratadas para este distúrbio. O grupo de mulheres que se submeteu ao tratamento para SPM apresentou mais sintomas relacionados à depressão, ansiedade e pensamentos negativos automáticos, do que o grupo que não se submeteu a tratamento para esta síndrome. Em pesquisas abordando este tema, de Barbenza e Almeyra (1997) encontraram correlação significativa entre SPM e neuroticismo, personalidades obsessivo-compulsivas, histeria e alto índice de ansiedade-traço; Hsiao, Liu, Chen e Hsieh (2002) observaram alto risco de outros transtornos psiquiátricos, especialmente os transtornos de humor.

A sociabilidade e a irritabilidade são características freqüentemente citadas nas pesquisas que tratam sobre SPM. As duas características foram examinadas por Marean, Fox, Cumming e Cumming (1998) em 20 mulheres com esta síndrome e em oito mulheres sem SPM (grupo controle), através do diário Daily Symptom Record Chart e das subescalas específicas do Multiscore Depression Inventory (The Social Introversion and Irritability Sub Escales). Os autores observaram que tanto os escores de irritabilidade quanto os de sociabilidade foram significativamente maiores no grupo com SPM, durante as fases pré-menstrual e menstrual.

$\mathrm{O}$ estresse também foi um dos enfoques de Woods e Lentz, Mitchell, Heikemper e Henker (1998), que realizaram estudo comparativo entre três grupos de mulheres, quarenta apresentando sintomas pré-menstruais com pouca intensidade, vinte e duas com síndrome pré-menstrual propriamente dita, e vinte e seis com manifestações exacerbadas desta síndrome. Estes pesquisadores avaliaram os fatores estimulantes e respostas a circunstâncias cotidianas geradoras de estresse e raiva, autocontrole, desencadeantes hormonais, susceptibilidade interpessoal. Verificaram que, no grupo com manifestações mais intensas de SPM, ocorreram vivências de experiências mais negativas, maior dificuldade em lidar com a raiva, e maior preocupação com o autocontrole do que o grupo com sintomas mais amenos. Durante o relato destas experiências foram percebidos sinais indiretos de respostas ao estresse como enrubescimento da face e tensão muscular. Contudo, as respostas cardiovasculares não acompanharam estes estressores. Os resultados levaram os autores a crer que, à medida que aumenta a idade das mulheres com SPM, mantêm-se as diferenças quanto resposta ao estresse e aos seus desencadeantes, quando comparadas com as mulheres com sintomas pré-menstruais amenos.

Cabe ressaltar, conforme refere Vilela, Souza e Rapelli (1996), a importância do diagnóstico diferencial entre quadros psiquiátricos, que têm intensificação de sintomas na 
fase lútea do ciclo menstrual, e manifestações psiquiátricas decorrentes da SPM. Para Pires e Calil (1999), são várias as evidências de que o período pré-menstrual está relacionado a maior vulnerabilidade para o agravamento de transtornos psiquiátricos preexistentes, havendo risco de piora, nesta fase específica do ciclo menstrual, de transtornos mentais crônicos, tais como bulimia, distimia, transtorno de ansiedade, abuso de substâncias, entre outros. Recomendam, a análise longitudinal ao longo do ciclo menstrual, através de diários e avaliações semanais, como estratégia para auxiliar a diferenciação entre a presença de disforia prémenstrual e a intensificação de condições psicopatológicas preexistentes.

\section{Método}

Um total de 609 universitárias, escolhidas aleatoriamente, de duas instituições particulares porto-alegrenses, com idades variando entre 18 e 35 anos, após receberem explicações sobre o objetivo da pesquisa, foram convidadas a preencher um questionário sobre cefaléias e sobre síndrome pré-menstrual, validado por Barea (1998), com pequenas modificações. Nesta triagem inicial, foram excluídas da pesquisa 248 mulheres que apresentavam algum tipo de patologia crônica associada, história de ciclos menstruais irregulares, ou que estavam utilizando alguma medicação que pudesse interferir nos critérios de avaliação da síndrome pré-menstrual. As 361 mulheres restantes foram convidadas a preencher o calendário para acompanhamento de SPM durante dois ciclos menstruais e, posteriormente, submeterem-se a participar da pesquisa. Deste grupo, 43 estudantes aceitaram participar desta fase da pesquisa, submetendo-se a uma entrevista individual para anamnese, exames físico e neurológico. Durante as entrevistas para anamnese, foram avaliadas as respostas dos questionários em comparação com as informações dos calendários, enfocando a existência ou não da SPM, de outra patologia associada, história de drogadição, utilização de alguma medicação naquele momento para o tratamento da síndrome, de alguma medicação que pudesse provocar ou interferir na SPM. Foi feita a aplicação coletiva do Z-Teste (Técnica de Zulliger) e do Inventário de Ansiedade Traço-Estado (IDATE) em dois grupos. Grupo 1, constituído de 25 mulheres com SPM, e Grupo 2, composto de 18 mulheres sem esta disfunção.

\section{Resultados}

Após a classificação, tabulação e planilhamento dos dados coletados, foi realizado estudo comparativo quanto às variáveis do Z-Teste e do IDATE entre o grupo de mulheres com SPM e o das mulheres sem SPM, no que tange às condições de personalidade, conforme segue. Foi realizado estudo comparativo entre os dois grupos de mulheres, usando o t-Teste para duas amostras independentes e estudo comparativo com nível de significância $\leq 0,05$. Seguem os resultados.

a) Investimento afetivo e reação emocional (Forma e Cor - FC; Cor e Forma - CF)

As respostas relacionadas ao determinante Forma e Cor (FC) expressam a capacidade de receber e retribuir afeto com adequação; a presença do determinante FC é um indicativo, no Z-Teste, de que a pessoa se permite ser objeto de inves- timento afetivo por parte das demais e, ao mesmo tempo, é capaz de investir os sentimentos afetivos adequadamente. Em outras palavras, é a capacidade de amar, estimar, ser amada e estimada. Já as respostas de C e CF são indicativos de que a pessoa tende a reagir afetiva e emocionalmente de forma não adequada, liberando a reação emocional sem se preocupar com as conseqüências.

Na comparação da média entre o Grupo 1 (de mulheres com SPM) e o Grupo 2 (de mulheres sem SPM) foi verificado que, para a variável CF, o nível de significância no estudo das diferenças entre os grupos foi de 0,01 (ver Tabela 1). Esta resultante indica que as mulheres do grupo com síndrome pré-menstrual reagem com alta intensidade e tendência à perda de controle aos estímulos emocionais. Seu sistema emocional se modifica com maior facilidade a determinados estímulos, sendo mais vulneráveis a escapes agressivos e a atitudes semidescontroladas, quando comparadas com o grupo das mulheres sem SPM.

Tabela 1. Quadro comparativo entre grupo de mulheres com SPM e grupo controle quanto aos determinantes Cor e Forma (CF), Cor (C), Sombreado perspectiva (FK, KF e K) Sombreado radiológico (Fk, kF, k), tratamento estatístico (t-Teste para duas mostras independentes)

\begin{tabular}{llcccc}
\hline & & N & Média & DP & Sig \\
\hline Espaço branco & SPM & 20 & 0,95 & 0,69 & \\
& Controle (12) & 18 & 0,72 & 0,67 & \\
\hline CF & SPM & 20 & 0,05 & 0,22 & \\
& Controle (12) & 18 & 0,22 & 0,43 & 0,01 \\
\hline \multirow{2}{*}{ Som C } & SPM & 20 & 0,00 & 0,00 & 0,03 \\
& Controle (12) & 18 & 0,05 & 0,24 & \\
\hline \multirow{2}{*}{ Som K } & SPM & 20 & 0,25 & 0,44 & \\
& Controle (12) & 18 & 0,22 & 0,55 & \\
\hline Som k & SPM & 20 & 0,10 & 0,31 & \\
& Controle (12) & 18 & 0,17 & 0,51 & \\
\hline
\end{tabular}

Outro resultado que chama a atenção é que o grupo 1, de mulheres com SPM, apresentou respostas caracterizadas pela Cor pura (Somatório de C), revelando maior descontrole do sistema afetivo-emocional do que as mulheres sem SPM $(p=0,03)$. Este determinante denota maior capacidade na expressão de "reações emocionais intensas e carregadas de sentimentos, quer de apreciação, estima, admiração, amor e paixão, quer de depreciação, desprezo, ódio, raiva e agressividade" (Vaz, 1997, p. 93).

b) Ansiedade traço e ansiedade estado (IDATE)

A inclusão do IDATE na pesquisa teve a finalidade de verificar se o grupo de mulheres com SPM apresentava indicativos de ansiedade como característica de personalidade (Traço) e ansiedade situacional (Estado).

Foi constatado para a variável Ansiedade-Estado no Grupo 1 (mulheres com SPM) o escore médio de 42,63, e no Grupo 2 (controle) 36,56. Para a outra variável Ansiedade-Traço o escore médio no grupo 1 (mulheres com SPM) foi de 47,58, e no grupo 2 (Controle) 40,83 (ver Tabela 2). $\mathrm{Na}$ comparação entre o grupo de mulheres com SPM e o grupo sem SPM (Controle), utilizando-se o t-Teste, não foi encontrada diferença significativa entre os dois grupos quanto à ansiedade situacional e à ansiedade como característica de personalidade. 
Tabela 2. Quadro comparativo entre grupo de mulheres com SPM e grupo controle (t-teste para duas amostras independentes) nos resultados do IDATE Não há diferença significativa entre os dois grupos

\begin{tabular}{lllccc}
\hline & Grupo & N & Média & DP & Sig \\
\hline Idate & SPM & 24 & 42,63 & 10,59 & 1.917 \\
Estado & Controle & 18 & 36,56 & 9,53 & 1.946 \\
\hline Idate & SPM & 24 & 47,58 & 9,60 & 2.000 \\
Traço & Controle & 18 & 40,83 & 12,29 & 1.931 \\
\hline
\end{tabular}

Discussão

Pode-se dizer que, de um modo geral, as mulheres com síndrome pré-menstrual e as mulheres sem esta síndrome, feita a comparação entre os dois grupos, utilizando o t-Teste, apresentaram algumas diferenças significativas em determinadas variáveis do Z-Teste. Verificamos, através da variável CF, que as mulheres do grupo com SPM reagem com intensidade e com tendência à perda de controle aos estímulos emocionais, com labilidade a escapes agressivos e a atitudes semidescontroladas quando comparadas com o grupo das mulheres sem SPM. Este resultado coincide com as considerações de Bancroft (1995) e de Halbreich (1997a) sobre a vulnerabilidade de determinadas mulheres a responder de forma emocionalmente desproporcional a agentes adversos. Isso corresponde ao aumento da ansiedade proporcionalmente à diminuição da atividade serotoninérgica, conforme Graeff (1999) a partir das teorias neurobiológicas do comportamento, assim como Epperson e cols.(2002), considerando a teoria da modulação GABAérgica do ciclo menstrual em mulheres com SPM e mulheres sem esta síndrome. É possível que flutuações na atividade serotonérgica durante o período pré-menstrual possam combinar-se com outras mudanças, tais como a dos hormônios gonadais (particularmente do estrógeno) e dos ritmos biológicos, contribuindo para o aparecimento de sintomas pré-menstruais em algumas mulheres.

A busca de explicações reducionistas aos sintomas emocionais da SPM, baseando-se exclusivamente em alterações hormonais nas mulheres com esta síndrome, tem redundado em resultados negativos, evidenciando que o aparecimento sintomatológico independe diretamente de desregulação hormonal. Contudo, a participação do ciclo menstrual tem sido fortalecida pelas comprovações de que a eliminação do ciclo ovariano pode resultar em melhora dos sintomas pré-menstruais.

A vulnerabilidade à SPM, no entender de Halbreich (2001), poderia ser entendida como consequiência da combinação entre modificações decorrentes da flutuação hormonal e circunstâncias externas, as quais não são propriamente papel do ciclo menstrual, mas que podem influir no modo como a mulher reagirá às mudanças hormonais ao longo do ciclo.

As labilidades a escapes agressivos e a atitudes semidescontroladas são sintomas pré-menstruais freqüentemente referidos nos consultórios clínicos e ginecológicos. A melhora significativa destes sintomas em resposta à utilização de medicamentos antidepressivos correlaciona-se positivamente com fatores biológicos interferindo nas condições emocionais destas mulheres. As ponderações feitas por Freeman e Rickels (1999) sobre a evolução satisfatória de mulheres que respondem a placebo, referindo supressão de seus sintomas, merecem atenção, sim. Entretanto, tais respostas ao placebo possivelmente se devam a mecanismos de reação defensiva, capazes de provocar somatização em mulheres que necessitam se adaptar a situação estressante em determinadas fases de suas vidas.

O expressivo indicativo de descontrole do sistema afetivo-emocional (Som C no Z-Teste), encontrado nas mulheres do grupo com SPM, permite inferir-se que as mulheres com SPM tendem a ter maiores dificuldades quanto às condições emocionais, quando comparadas com as mulheres sem estes sintomas. Estes dados, embora colhidos de amostra reduzida, se aproximam dos resultados das pesquisas realizadas por Van den Akker e cols. (1995) e Barbenza e Almeyra (1997), que encontraram correlação significativa entre SPM e neuroticismo; por Bloch, Schmidt e Rubinow (1997) que observaram irritabilidade e labilidade emocional nestas mulheres, e por Woods e cols. (1998) que identificaram nas mulheres com SPM dificuldades em lidar com o sentimento de raiva e maior preocupação com o autocontrole do que em mulheres sem esta síndrome.

Ainda são poucas, as publicações específicas sobre as condições emocionais de mulheres que sofrem de síndrome pré-menstrual. Um dos motivos relacionados a esta insuficiência de produção científica possivelmente se deve à percepção biológica do médico em geral sobre esta síndrome.

\section{Conclusões}

Alguns autores têm identificado índices aumentados de neuroticismo, irritabilidade, ansiedade e depressão em mulheres que sofrem de síndrome pré-menstrual. Através do Z-Teste, constatou-se em mulheres adultas jovens com SPM, a presença de indicativos de reação emocional intensa, tendência à perda de controle e a atitudes semidescontroladas, escapes agressivos e maior descontrole do sistema afetivo-emocional do que grupo das mulheres sem SPM. As diferenças encontradas entre os dois grupos de mulheres da amostra, embora significativas, necessitam de estudo em amostras maiores e diversificadas, notadamente quanto às faixas etárias, para que se possa afirmar que tendência à perda de controle, atitudes semidescontroladas e escapes agressivos sejam peculiares à mulher que sofre de SPM.

\section{Referências}

Bancroft, J. (1995). The menstrual cycle and the well being of women. Social Science Medicine, 41 (6), 785-791.

Barbenza, C.M.\& Almeyra, I. (1997). Perfil psicologico de un grupo de mujeres que presentan el sindrome premenstrual. Revista de Psiquiatria de la Facultad de Medicina de Barcelona, 24 (5), 126-132.

Barea, L.M. (1998). An epidemiologic study of headache among children and adolescents of southern Brazil. Cephalalgia, 16, 545 - 550 .

Barnhart, K.T., Freeman, E.W. \& Sondheimer, S.J. (1995). A clinician's guide to the premenstrual syndrome. Medicine Clinics of North America, 79 (6), 1457-1472.

Bhatia, S.C. \& Bhatia, S. K. (1999). Depression in women: diagnostic and treatment considerations. American Family Physician, 60 (1), 225-234. 
Bloch, M., Schmidt, P.J. \& Rubinow, D.R. (1997). Premenstrual syndrome: evidence for symptom stability across cycles. American Journal of Psychiatry, 154 (12), 1741-1746.

Cahill, C.A. (1998). Differences in cortisol, a stress hormone, in women with turmoil-type premenstrual symptoms. Nursing Research, 47 (5), 278-284.

Chau, J.P.C. \& Chang, A. M. (1998). Relationship between premenstrual tension syndrome and anxiety in Chinese adolescents. Journal of Adolescent Health, 22 (3), 247-249.

Choi, P.Y. \& McKeown, S. (1997). What are young undergraduate women's qualitative experiences of the menstrual cycle? Journal of Psychosomatic Obstetric Gynaecology, 18 (4), 259-265.

Condon, J.T. (1993). The premenstrual syndrome: a twin study. British Journal of Psychiatry, 162, 481-486.

Christensen, A.P. \& Oei, T.P. (1995). Correlates of premenstrual dysphoria in help-seeking women. Journal of Affective Disorders, 33 (1), 47-55.

Daugherty, J.E. (1998). Treatment strategies for premenstrual syndrome. American Family Physician, 58 (1), 183-192.

Epperson, C.N., Haga, K., Mason, G.F., Sellers, E., Gueorguieva, R., Zhang, W., Weiss, E., Rothman, D.L. \& Krystal, J.H. (2002). Cortical gamma-aminobutyric acid levels across the menstrual cycle in healthy women and those with premenstrual dysphoric disorder: a proton magnetic resonance spectroscopy study. Archives of General Psychiatry, 59 (9), 851-858.

Facchinetti, F., Tarabusi, M. \& Nappi, G. (1998). Premenstrual syndrome and anxiety disorder: A psychobiological link. Psychotherapy and Psychosomatics, 67 (2), 57-60.

Freeman, E.W., Rickels, K., Schweizer, E. \& Ting, T. (1995). Relationships between age and symptom severity among women seeking medical treatment for premenstrual symptoms. Psychological Medicine, 25 (2), 309-315.

Freeman, E.W. \& Rickels, K. (1999). Characteristics of placebo responses in medical treatment of premenstrual syndrome. American Journal of Psychiatry, 156 (9), 1403-1408.

Glick, H., Endicott, J. \& Nee, J. (1993). Premenstrual changes: are they familial? Acta Psychiatrica Scandinavica, 88, 149-155.

Graeff, F.G. (1999). Ansiedade. Em F.G. Graeff \& M.L Brandão (Orgs.), Neurobiologia das Doenças Mentais (pp. 135-178). São Paulo: Lemos Editorial.

Gurevich, M. (1995). Rethinking the label: who benefits from the PMS construct? Women \& Health, 23 (2), 67-98.

Halbe, H.W. (1987). Síndrome da tensão pré-menstrual. Tratado de Ginecologia, 2. São Paulo: Editora Roca.

Halbreich U. (1995). Menstrually related disorders: what we do know, what we only believe that we know, and what we know that we do not know. Critical Reviews in Neurobiology, 9, 163-175.

Halbreich, U. (1997a). Premenstrual dysphoric disorders: a diversified cluster of vulnerability traits to depression. Acta Psychiatrica Scandinavica, 95 (3), 169-176.

Halbreich, U. (1997b). Menstrually related disorders towards interdisciplinary international diagnostic criteria. Cephalalgia, 17 (20), 1-4.

Halbreich, U. \& Kahn, L.S. (2001). Role of estrogen in the aetiology and treatment of mood disorders. Drugs, 15 (10), 797-817.

Hsiao, M.C., Liu, C.Y., Chen, K.C. \& Hsieh, T.T. (2002). Characteristics of women seeking treatment for premenstrual syndrome in Taiwan. Acta Psychiatrica Scandinavica, 106 (2), 150-5.
Kendler, K.S., Karkowski, L.M., Corey, L.A. \& Neale, M.C. (1998). Longitudinal population-based twin study of retrospectively reported premenstrual symptoms and lifetime major depression. American Journal of Psychiatry, 155 (9): 1234-1240.

Lima, C. A.\& Camus, V. (1996). Síndrome pré-menstrual: um sofrimento ao feminino. Psiquiatria Biológica, 4 (3),137-146.

Marean, M., Fox, E., Cumming, C.E. \& Cumming, D.C. (1998). Irritability and sociability in women with symptomatic premenstrual change. Women \& Health, 27 (3), 65-71.

Melo, N.R., Giribela, A.H.G., Giribela, C.R. G.\& Ricci, M.D. (2001). Síndrome pré-menstrual. Revista Brasileira de Medicina, 58, 185-192.

Mortola, J.F. (1992). Issues in the diagnosis and research of premenstrual syndrome. Clinical Obstetrics and Gynecology, 35 (3), 587-598.

Nogueira, C.W.M. \& Silva, J.L.P. (2000). Prevalência dos sintomas da síndrome pré-menstrual. Revista Brasileira de Ginecologia e Obstetrícia, 22 (6), 347-351.

O'Brien, P.M. (1997). Clinical concerns with premenstrual syndrome. Cephalalgia, 17 (Suppl 20), 1-8.

Pearlstein, T.B. (1995). Hormones and depression: what are the facts about premenstrual syndrome, menopause, and hormone replacement therapy? American Journal of Obstetrics and Gynecology, 173 (2), 646-653.

Pires, M.L.N. \& Calil, H.M. (1999). Associação entre transtorno disfórico pré-menstrual e transtornos depressivos. Revista Brasileira de Psiquiatria, 21 (2), 118-127.

Reid, R.L. \& Yen, S.S.C. (1983). The premenstrual syndrome. Clinical Obstetrics and Gynecology, 26, 710.

Richardson, J.T. (1995). The premenstrual syndrome: a brief history. Social Science \& Medicine, 41 (6), 761-767.

Rosales, C.L., Guerrero, J.A.A. \& Moreno, L.G. (1996). Tratamiento del sindrome de tension premenstrual (STP) con maleato de lisurida. Ginecología y Obstetricia de Mexico, 64, 556-560.

Severino, S.K., Moline, M.L. (1995). Premenstrual syndrome. Identification and management. Drugs, 49 (1), 71-82.

Spielberger, C.D., Gorsuch, R.L. \& Lushene, R.E. (1979). Inventário de Ansiedade Traço-Estado. Rio de Janeiro: CEPA.

Sveinsdottir, H. (1998). Prospective assessment of menstrual and premenstrual experiences of Icelandic women. Health Care for Women International, 19 (1), 71-82.

Van den Akker, O.B., Eves, F.F., Stein, G.S. \& Murray, R.M. (1995). Genetic and environmental factors in premenstrual symptom reporting and its relationship to depression and a general neuroticism trait. Journal of Psychosomatic Research, 39 (4), 477-487.

Van den Akker, O.B., Eves, F.F., Service, S. Lennon, B. (1995). Menstrual cycle symptom reporting in three British ethnic groups. Social Science \& Medicine, 40 (10), 1417-1423.

Vaz, C.E. (1997). O Rorschach teoria e desempenho, (3 ${ }^{\mathrm{a}}$ ed). São Paulo: Editora Manole Ltda.

Verri, A., Nappi, R.E., Vallero E., Galli, C., Sances, G. \& Martignoni, E. (1997). Premenstrual dysphoric disorder and eating disorders. Cephalalgia, 17 (suppl. 20), 25-28.

Vilela, M.S.B., Souza, L.M.B.\& Rapelli, C.B. (1996). Manifestações psiquiátricas da síndrome pré-menstrual: revisão de literatura Revista de Ciências Médicas - PUCCAMP, 5 (1), 30-3.

Walker, A. (1995). Theory and methodology in premenstrual syndrome research. Social Science \& Medicine, 41 (6), 793-800. 
Woods, N.F, Lentz, M.J. Mitchell, E.S., Heitkemper, M.S., J. \& Henker, R. (1998). Perceived stress, physiologic stress arousal, and premenstrual symptoms: group differences and intra-individual patterns. Research in Nursing \& Health, 21 (6), 511-523.

Wyatt, K.M., Dimmock, P.W., Frischer, M., Jones, P.W., O’Brien, S.P. (2002). Prescribing patterns in premenstrual syndrome. BioMed Central Women's Health, 2 (1), 4.

Wyatt, K.M., Dimmock, P.W., Jones, P., Obhrai, M., O’Brien, S. (2001). Efficacy of progesterone and progestogens in manage- ment of premenstrual syndrome: systematic review. British Medical Journal, 6 (323), 776-780.

Yonkers, K.A. \& Chantilis, S.J. (1995). Recognition of depression in obstetric/gynecology practices. American Journal of Obstetrics and Gynecology, 173, (2), 632-638.

Yu, M., Zhu, X. Li, J., Oakley, D. \& Reame, N., E. (1996). Perimenstrual symptoms among chinese women in an urban area of China. Health Care for Women International, 17 (2), 161172. 


\section{Princípios Normativos Para Publicação em Psicologia: Teoria e Pesquisa}

\section{Encaminhamento de manuscrito para publicação}

Ao submeter um manuscrito para publicação, o autor deve incluir uma carta endereçada ao editor, indicando seu interesse na publicação do trabalho em Psicologia: Teoria e Pesquisa.

A carta deve conter informações gerais sobre o manuscrito, por exemplo, em qual categoria de contribuição o autor classifica seu trabalho, se o manuscrito foi preparado a partir de uma dissertação ou tese (informar nome de orientador e co-orientador(es), instituição, banca examinadora), se corresponde a texto apresentado em um encontro científico (informar quando e onde). Essas e outras informações recomendadas aqui são úteis ao editor, por exemplo, para a verificação do cumprimento das normas de publicação, e para a escolha da consultoria. (Sobre escolha da consultoria ver, p. ex., o editorial de Feitosa, 1993, Vol. 9, n. 3, pp. iv-vi).

No caso de autoria múltipla, espera-se que todos os autores estejam de acordo com a apresentação do manuscrito para publicação. Recomenda-se que o encaminhamento seja feito com a assinatura de todos os autores, certificando seu interesse na publicação do trabalho em Psicologia: Teoria e Pesquisa.

No caso de autoria múltipla, deve ser indicado o autor responsável pela correspondência com o editor. Esse autor será considerado o autor principal para fins de comunicação sobre o manuscrito.

Se for o caso, é na apresentação do manuscrito que o autor notifica a existência de outros trabalhos seus, de conteúdo relacionado, já publicados ou submetidos simultaneamente a esta ou a outra revista.

Se o manuscrito contém reprodução de partes de trabalho já publicado, recomenda-se que ao submeter o manuscrito o autor já inclua a documentação exigida, conforme o caso (permissão escrita ou comprovação de direito de reprodução). $\mathrm{O}$ autor deveria notificar qualquer pendência de copyright, se fosse o caso.

Em sua maior parte, o conteúdo deste texto foi traduzido e adaptado do Manual de Publicação da APA (APA, 1994). 